\title{
Maintaining Ethical Approach in Rare Diseases in Bulgaria
}

\author{
Mariela Deliverska ${ }^{1}$ \\ ${ }^{1}$ Associate Professor, Ph.D, Department of "Medical Ethics and Law", Faculty of Public Health, Medical \\ University - Sofia, Bulgaria
}

\begin{abstract}
Ethical approach towards rare diseases should be introduced at highest possible level in every national framework, as it is directly related to the right to the highest attainable standard of health. Although most rare diseases are genetic diseases, they can also result from environmental exposures during pregnancy or later in life, often in combination with genetic susceptibility. Some are rare forms or rare complications of other common diseases. The main problems posed by rare diseases defined at European Union level have focused the attention to some ethical consequences raised by the lack of recognition and visibility of rare diseases; plus the lack of detailed strategies for rare diseases; and the lack of European cooperation, coordination, and regulation for rare diseases. In Bulgaria, the national authorities are still taking actions to ensure the proper recognition and visibility of rare diseases. In Bulgaria, the National registry of patients with rare diseases is established and maintained by the National Center of Public Health and Analyses.Bioethics Mediation in daily clinical practice. Bioethics Mediation will be patientoriented and respect the principles and values of both of the two sides of healthcare.
\end{abstract}

Key Words:rare diseases; diagnosis, quality, healthcare services, commission on rare diseases

\section{INTRODUCTION}

Rare diseases are a threat to the health of patients as they are life threatening or chronically debilitating diseases with a low prevalence and a high level of complexity. Despite their rarity, there are so many different types of rare diseases that millions of people are affected. Because of their low prevalence, their specificity and the high total number of people affected, rare diseases call for a global approach based on special and combined efforts to prevent significant morbidity or avoidable premature mortality, and to improve the quality of life and socioeconomic potential of affected persons.

Although most rare diseases are genetic diseases, they can also result from environmental exposures during pregnancy or later in life, often in combination with genetic susceptibility. Some are rare forms or rare complications of other common diseases.

Some rare diseases have prevalence near the threshold of 5 per 10,000, such as Gelineau disease, triple X syndrome, scleroderma or neural tube defects. Most rare diseases are very rare, affecting one in 100,000 people or less, such as Gaucher disease, Ewing sarcoma, Duchenne muscular dystrophy, or Von Hippel-Lindau disease.

There is also a great diversity in the age at which the first symptoms occur; half of the rare diseases can appear at birth or during childhood (such as Williams' syndrome, Prader-Willi 
syndrome, and retinoblastoma). The other half of rare diseases can appear in adulthood (such as Huntington's disease, Creutzfeld-Jacob disease, amyotrophic lateral sclerosis).

Several years ago, the main problems posed by rare diseases defined at European Union level focused the attention to some ethical consequences raised by the lack of recognition and visibility of rare diseases; a lack of detailed strategies for rare diseases; and a lack of European cooperation, coordination, and regulation for rare diseases.

The lack of formal identification in health systems imposes medical and financial barriers in receiving treatment for an unrecognized disease. This does not allow achieving a significant improvement of life-quality for thousands of rare disease patients.

The difference in availability and quality of care provided to patients with rare diseases by the national healthcare services for diagnosis, treatment, and rehabilitation decreases the chances of achieving significant prolongation of life of patients with rare diseases. Patients often face lack of quality information and appropriate support at the time of diagnosis, as well as lack of appropriate multidisciplinary healthcare, which results in a devaluation of the main principles in health systems such as universality, access to good quality care, equity and solidarity.

Without correct diagnoses, people with rare diseases are not considered rare disease patients and are thus restricted in their ability to seek appropriate medical attention and social assistance in their respective healthcare systems.

According to the reference portal for information on rare diseases and orphan drugs Orphanet, of the thousands of known rare diseases for which a clinical identification is possible, only 250 of them have a code in the existing International Classification of Diseases (ICD) (10th version). An appropriate classification and codification of all rare diseases is necessary in order to give them the necessary visibility and recognition in national health systems. The ICD is being revised to better reflect progress in health sciences and medical practice. In line with advances in information technology, ICD-11 will be used with electronic health applications and information systems. The upcoming World Health Organization's (WHO) 11th International Statistical Classification of Diseases and Related Health Problems (ICD-11) is expected to be finalized in 2018.

The ICD is important because it provides a common language for reporting and monitoring diseases. This allows the world to compare and share data in a consistent and standard way between hospitals, regions and countries and over periods of time. It facilitates the collection and storage of data for analysis and evidence-based decision-making. WHO has established various Topic Advisory Groups to serve as planning and advisory bodies in the update and revision process for specific areas.

The production of basic information on which to build the classification of rare diseases in ICD11 was assigned to Orphanet. It contributed to the whole ICD revision process, considering that rare diseases involve all areas of medicine. Orphanet collects series of rare diseases classifications mainly based on scientific grounds (etiology and mechanism). To complement these classifications, a clinical in-house classification is developed to meet the needs of the clinicians. All the classifications, regularly updated as scientific knowledge evolves, can be viewed on the Orphanet website and have served as a basis to build ICD-11.

Even though many affected individuals live full and happy lives and may not experience pain or suffering, many families remain profoundly affected by genetic conditions, in spite of improved treatment, education, and support services. There is a substantial cost to society for noninstitutional, outpatient, educational, medical and social services, as well as lost economic output from family members who care for persons with genetic disorders.

Genetic conditions occur with similar frequencies in different nations and irrespective of the socio-economic status of individuals. A meaningful right to health care must include access to services for the diagnosis, treatment, and prevention of genetic disorders. The priority assigned to 
genetic services with respect to other health services is a matter of public health policy in each country.

Within genetic services, efforts should be directed towards extending the reach of genetic services at the primary care level, with the utilization of technologies and personnel that are appropriate to the needs, expectations, and beliefs of the community.

The difficulty in obtaining the correct diagnosis is one of the most important issues for rare disease patients, because it may take years or even decades before a final diagnosis is obtained. Late diagnoses delay the beginning of adapted treatments and can have severe irreversible, debilitating and life-threatening consequences. When seeking diagnosis, patients frequently consult numerous doctors, undergo multiple examinations and often receive various incorrect diagnoses resulting in inefficient and even harmful treatments.

The individual consequences of improper diagnosis include the worsening in clinical status, plus psychological damage often related to medical denial of the undiagnosed disease and, in some cases, death.

Another important issue, which has an ethical approach, is the lack of good clinical practice guidelines for some of the existing rare disease conditions. This is mainly caused by the segmentation of medical specialities and lack of application of a multidisciplinary approach to care for patients suffering from a rare disease.

In an attempt to solve the difficulties faced by patients with rare diseases, at the European Union level there has been introduced the development of European reference networks (ERNs) for rare diseases. These networks should serve as research and knowledge centres, updating and contributing to the latest scientific findings, treating patients from Member States and ensuring the availability of subsequent treatment facilities where necessary.

In Bulgaria, the national authorities are still taking actions to ensure the proper recognition and visibility of rare diseases.

In 2014 Bulgarian authorities adopted the Ordinance on the conditions and procedures for registration of rare diseases and designation of the Centres of Expertise and Reference Networks for rare diseases. This act contains the main provisions for the establishment of a Commission on rare diseases; on the consistency of a List of rare diseases and the establishment of National registry for rare diseases. The Ordinance also sets out specific rules and procedures for designating Centres of Expertise for rare diseases [1].

The Bulgarian Commission on rare diseases consists of a total of 16 members, of whom 7 are medical specialists and 4 representatives of the Ministry of Health. It supports the activities of the Minister of Health and the Director of the National Center of Public Health and Analyses (NCPHA) on matters concerning rare diseases. The National Center of Public Health and Analyses is a structure within the national healthcare system, which is responsible for carrying out activities for protecting public health, promoting health and preventing diseases, and providing information for healthcare management.

Additional competences assigned to the Bulgarian Commission on rare diseases include advice to the Minister of Health in relation to his competences and issuing opinions on proposals for inclusion of diseases on the list of rare diseases, as well as assessment of the activities of the National Registry of patients with rare diseases and assessment of Centres of Expertise and Reference Networks functioning in Bulgaria.

The List of rare diseases is mainly used:

1. in the procedures for designation of Centers of expertise and Reference networks for rare diseases;

2. in relation to the National Register of patients with rare diseases;

3. for the development of medical science and practice; 
4. when planning diagnostic activities, treatment, monitoring and rehabilitation of rare diseases;

5. for the establishment of European and international cooperation in exchange of information and scientific experience when providing medical care of patients with rare diseases.

The List of rare diseases is introduces based on a rare disease dossier, which contains specific data such as: name, definition and code (ICD and ORPHAcode) of the disease; epidemiological data for Bulgaria and the European Union, compliance with the official definition for rare disease; diagnostic criteria and guidelines; treatment, follow up and rehabilitation guidelines; prevention guidelines (if applicable); proposals and recommendations for organization, funding and management of the medical services for the diseases in question; description of experience with specific patients with the disease in question (if available).

The specifics of rare diseases create challenges for the registration of patients. The genetic nature of most rare diseases suggests the need to investigate and track family related cases, which is not always possible. The combination of a small number of cases and a large geographic scope of data collection requires multiple collaborations and exchange of information, usually at international level, often constrained by legal frameworks. The need for resources to create and maintain registries for rare diseases often puts into delay the introduction of such database.

In Bulgaria, the National registry of patients with rare diseases is established and maintained by the National Center of Public Health and Analyses. All medical treatment facilities in Bulgaria, including the Centres of Expertise, are mandated to submit epidemiological data to the Registry, as the data should cover all rare conditions that are present in the List of Rare Diseases. The NCPHA is required to prepare and publish annual reports on the epidemiology of rare diseases in Bulgaria. These reports include information contained at the National registry of patients with rare diseases, as well as on diseases dossiers.

In Bulgaria, the NCPHA is the competent authority leading, controlling, monitoring and coordinating information activities in the healthcare, as it unifies medical and statistical documentation for the health status of the population. It is also responsible for development, implementing and maintaining national standards of coding in hospitals. Its activity also directly reflects to the conditions at the healthcare system in relation to rare conditions.

An ethical approach towards rare diseases should be introduced at highest possible level in every national framework, as it is directly related to the right to the highest attainable standard of health. Health care and treatment for rare diseases should meet the requirements of criteria providing better access to consultations and medical exams, multidisciplinary approach in preventing misdiagnosis of rare diseases, as well as psychological treatment and support in cases of misdiagnosis. In stages of prevention, research and treatment, patients should be provided with good information quality and proper conditions for autonomous decision-making, as this will guarantee the exercise of the main human right to health.

\section{RECOMMEDNDED READING AND REFERENCES}

1. Commission of the European Union, Commission staff working document accompanying the Commission from the Commission to the European Parliament, the Council, the Economic and Social Committee and the Committee of the Regions on Rare Diseases: Europe's challenges and the Proposal for a Council recommendation on a European actions in the field of rare diseases Impact assessment, SEC 2712, 11.11.2008

2. Council of the European Union, Council Recommendation on an action in the field of rare diseases, OJ C151, 3.7.2009

3. Ministry of Health, National Center of Public Health and Analyses, Annual report on the activities for 2015 . 
4. Ministry of Health, Ordinance No. 16 of 30 July 2014 of the Terms and Conditions for registration of rare diseases and Centres of Expertise and Reference Networks for rare diseases., State Gazette No. 67, 12 August 2014.

5. Miteva-Katrandzhieva TS. Overview of epidemiological rare diseases in Bulgaria, Rare Dis Orph Drugs 2016;3(1):11-5.

6. Orphanet, List of rare diseases and synonyms listed in alphabetical order.-Orphanet Report Series, Rare Diseases collection, 2016

7. Orphanet, Prevalence and incidence of rare diseases: Bibliographic data.- Orphanet Report Series, Rare Diseases collection, March 2016.

Acknowledgements - Nil

Source of Funding - Nil

Conflict of Interest - Nil 